\title{
FORMACIÓN DE LOS JÓVENES UNIVERSITARIOS Y PERCEPCIÓN DE LA VIOLENCIA.
}

\section{ELISA LARRAÑAGA}

SANTIAGO YUBERO

Escuela Universitaria de Trabajo Social. Cuenca. Universidad de Castilla-La Mancha.

\section{INTRODUCCIÓN.}

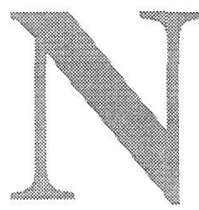

o podemos olvidar que nuestros alumnos trabajan en las dos aceras del problema de la violencia, con las personas que han sufrido violencia, pero también con las personas que la han suministrado, con las personas violentas.

Socialmente las actitudes ante las personas que han sufrido la violencia son muy positivas y abiertas, con potenciación de ayuda y comprensión. Nuestro centro de análisis se sitúa en la otra cara, en el posicionamiento ante las personas que generan la violencia.

Nuestro objetivo es conocer la opinión de los universitarios y analizar las peculiaridades de los alumnos de Trabajo Social en el campo que nos ocupa: actitudes ante la persona violenta. Queremos valorar la categorización inmediata, de forma automática, que realizan los estudiantes universitarios ante la persona violenta.

Para ello, construimos un cuestionario sobre:

- Atribuciones que realizan sobre los actos de violencia.

- Representaciones sociales de la persona violenta.

- Actitud ante la persona violenta, valorando sus tres componentes: cognitivo, afectivo y conductual.

El cuestionario fue complementado por 200 estudiantes, durante el primer cuatrimestre de este curso académico, procedentes de tres Escuelas Universitarias de la Universidad de Castilla-La Mancha, del campus de Cuenca. Las muestras son:

- Trabajo Social: 88 alumnos (66 mujeres y 22 varones).

- Enfermería: 62 alumnos (51 mujeres y 11 varones).

- Politécnica: 50 alumnos (14 mujeres y 36 varones).

Un planteamiento cada día más contradictorio de la enseñanza uni- 
versitaria es si los estudios de una carrera deben prepararte, fundamentalmente, en una serie de técnicas para una incorporación satisfactoria al mundo del trabajo una vez terminados los estudios y dejar en un segundo plano la formación humana de los estudiantes en concordancia con el perfil de la carrera elegida; o bien unos estudios que, sin dejar de lado el perfil profesional, hagan hincapié en la preparación cognitiva del sujeto, en cuanto actitudes, valores sociales, creencias..., con respecto a las intervenciones sociales propias de la profesión elegida. En este sentido, en función de los estudios, o por el efecto de cursarlos, unos estudiantes deben estar más sensibilizados que otros, con respecto a determinado tema.

Así, consideramos que en cuanto al tema de la violencia, los estudiantes de Trabajo Social, futuros profesionales, deben conocer más matices que otros y tener una sensibilidad social que vaya en la línea del papel del rol social del trabajador social.

La elección de estudiantes de carreras tan distintas, a priori, nos podría permitir comparar el "enfoque educativo" de las distintas carreras.

Queremos ir más allá de la simple comparación inter-centros y analizar cuál puede ser el origen de las diferencias que aparezcan: ¿es por la formación que reciben en la E.U.T.S.? o ¿previamente existen diferencias entre los estudiantes?

Para poder responder a estas preguntas realizaremos dos contrastes complementarios:

$1{ }^{\circ}$ Comparaciones entre las tres muestras, Trabajo Social-Enfermería-Politécnica.

Entendiendo que si no aparecen diferencias, posiblemente la valoración del objeto evaluado responde a la influencia del contexto sociocultural común en el que están insertos culturalmente los sujetos, siendo sus procesos de aprendizaje social, en este tema homogéneos.

$\mathrm{Si}$ aparecen diferencias entre grupos, pasaremos a la realización del segundo contraste.

2. ${ }^{\circ}$ Comparación de los alumnos de Trabajo Social, segmentando los alumnos de primer y tercer curso, con el resto de los estudiantes. Infiriendo que:

- Si las diferencias se producen con los alumnos de tercer curso, su origen podrá ser debido a la formación específica que reciben en Trabajo Social, formación curricular.

- Si las diferencias aparecen ya con los alumnos de primer curso no puede ser debido al componente de formación curricular, pues acaban de iniciar su andadura en la Escuela. Serían, por tanto, 
diferencias debidas a condiciones personales previas, características de grupo de pertenencia de los individuos.

\section{RESULTADOS.}

\subsection{Atribuciones.}

Las atribuciones son inferencias que hacemos las personas sobre las causas de una conducta. Son fundamentalmente sociales, en el sentido de que la atribución subraya las creencias compartidas culturalmente.

\section{Los resultados obtenidos han sido:}

Tabla 1: Razones para que las personas se comporten violentamente (en \%).

\begin{tabular}{|l|c|c|c|}
\hline & Trabajo Social & Enfermería & Politécnica \\
\hline Sentirse a disgusto en esta sociedad. & 45.5 & 38.7 & 32.0 \\
\hline Por el gusto de hacer algo antisocial. & 12.5 & 19.4 & 18.0 \\
\hline Para calmar los nervios. & 15.9 & 21.0 & 8.0 \\
\hline Por diversión. & 9.1 & 16.1 & 16.0 \\
\hline Porque lo hacen los amigos. & 17.0 & 22.6 & 12.0 \\
\hline Porque tiene dificultades en el trabajo. & 4.5 & 3.2 & 4.0 \\
\hline Porque tiene dificultades en la familia. & 14.8 & 12.9 & 14.0 \\
\hline Por inseguridad. & 47.7 & 32.3 & 40.0 \\
\hline Otras. & 15.9 & 21.0 & 22.0 \\
\hline NS/NC. & 1.1 & 3.2 & 4.0 \\
\hline
\end{tabular}

Gráfica 1:

Atribuciones sobre el comportamiento violento.

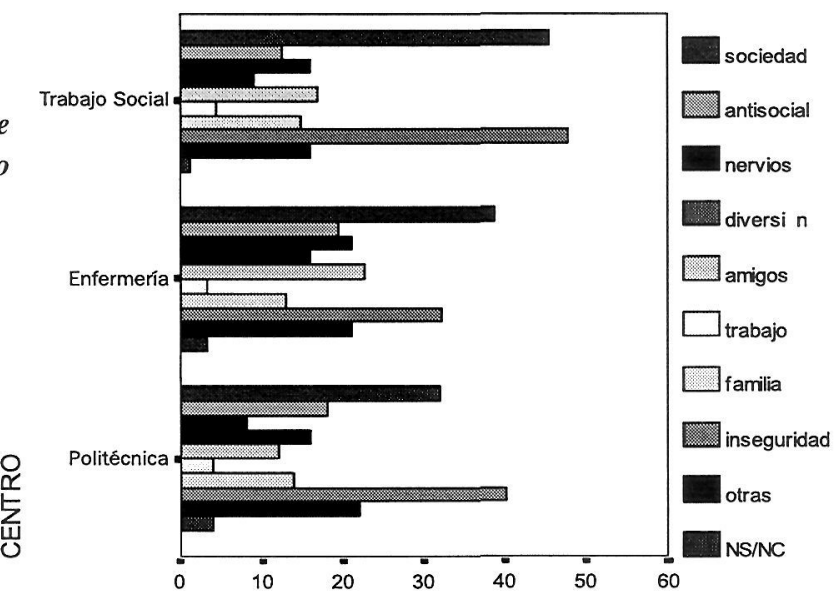

Suma 
En la gráfica 1 se aprecia claramente el predominio de las dos mismas respuestas en los tres grupos de estudiantes: "sentirse a disgusto en esta sociedad" e "inseguridad". En ambos casos los porcentajes de respuesta son ligeramente más altos en los alumnos de Trabajo Social (tabla 1), grupo en el que también es un poco más bajo el porcentaje de respuesta de diversión. En general, supone una gran homogeneidad de respuesta, lo que implica la confirmación del carácter social de las atribuciones.

Si analizamos las respuestas con la muestra de Trabajo Social segmentadas las diferencias en porcentajes de respuesta se mantienen con respecto al resto de los grupos. Los porcentajes alcanzados son:

- Sentirse a disgusto en esta sociedad: $1^{\circ}-44.4 \%, 3^{\circ}-46.5 \%$, frente a un $35.7 \%$ de los demás.

- Por inseguridad: $1^{\circ}-46.7 \%, 3^{\circ}-48.8 \%$, frente a un $35.7 \%$ de los demás.

La igualdad entre $1^{\circ}$ y $3^{\circ}$ implicaría que se trata además de un planteamiento previo de los alumnos que han elegido cursar Trabajo Social, presentando una mayor homogeneidad de respuesta, se podría interpretar como una característica de grupo.

La respuesta "por el gusto de hacer algo antisocial" es más reducida en el grupo de alumnos de Trabajo Social. Analizada esta categoría de respuesta de forma segmentada se observa que la diferencia se produce en los alumnos de tercer curso, los alumnos de primer curso alcanzan igual porcentaje de respuesta que los demás (17.8\% y $18.8 \%$ respectivamente, frente a un $7 \%$ en tercero). Lo que supondría que la formación curricular recibida como trabajadores sociales lleva a la reducción de esta respuesta.

\subsection{Representaciones sociales.}

Las personas somos portadores de un conjunto de representaciones sociales que nos disponen en la forma de analizar y evaluar las situaciones y que pueden determinar la conducta social que se lleve a cabo. En el proceso de formación vamos abstrayendo de nuestras experiencias y/o de la información que recibimos, estructuras de conocimiento que reflejan las asociaciones entre atributos que se dan en la realidad social. Pero no es una mera adicción de elementos aislados, es el producto de una actividad constructiva guiada por las ideas de la persona.

Los resultados obtenidos han sido: 
Tabla 2: Características que mejor califican a una persona violenta (en \%).

\begin{tabular}{|l|r|r|c|}
\hline & Trabajo Social & Enfermería & Politécnica \\
\hline Inconformista & 18.2 & 12.9 & 14.0 \\
\hline Infeliz & 51.1 & 66.1 & 48.0 \\
\hline Enfermo & 53.4 & 59.7 & 48.0 \\
\hline Divertido & 0 & 0 & 6.0 \\
\hline Marginado & 14.8 & 9.7 & 12.0 \\
\hline Moderno & 1.1 & 0 & 2.0 \\
\hline Valiente & 4.5 & 0 & 8.0 \\
\hline Víctima & 21.6 & 1.6 & 10.0 \\
\hline Liberado & 1.1 & 1.6 & 2.0 \\
\hline Delincuente & 27.3 & 45.2 & 40.0 \\
\hline
\end{tabular}

En la tabla 2 aparecen los porcentajes de respuesta sobre los diez adjetivos presentados para describir a una persona violenta. Puede apreciarse un predominio de la semejanza de la distribución excepto en dos categorías: "delincuente" y "víctima". En las tres muestras los porcentajes más elevados son los correspondientes a "enfermo" e "infeliz", en tercer lugar "delincuente", pero con una importante diferencia en porcentaje de respuesta entre los alumnos de Trabajo Social y los demás estudiantes. En siguiente lugar se produce coincidencia entre Enfermería y Politécnica, en el adjetivo "inconformista", frente a "víctima" para los estudiantes de Trabajo Social (representando el 76\% de los sujetos que marcan esta diferencia). En las gráficas 2 y 3 aparecen reflejados los resultados de cada una de las muestras en estos adjetivos.

Gráfica 2: Porcentajes de respuesta en adjetivos más frecuentes.

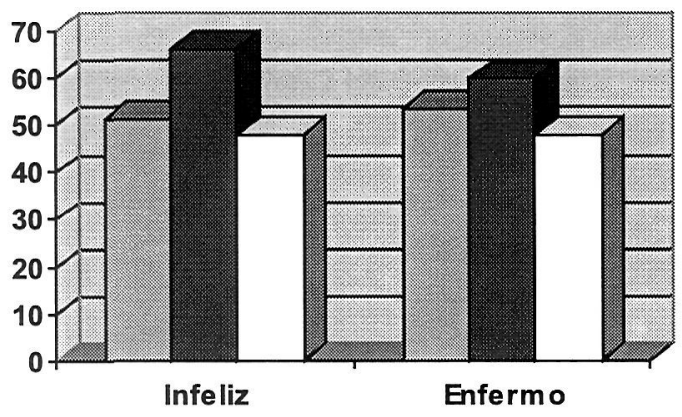

$\square$ Trabajo Social
Enfermería
$\square$ Politécnica


Gráfica 3: Porcentajes de respuesta en adjetivos diferenciales.

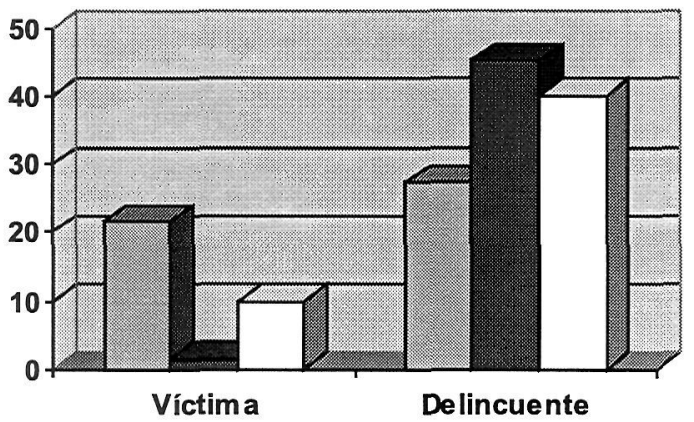

\begin{tabular}{|l}
\hline Trabajo Social \\
Enfermería \\
$\square$ Politécnica
\end{tabular}

La imagen prototípica de la persona violenta para cada una de las muestras es:

\begin{tabular}{|l|l|l|}
\hline TRABAJO SOCIAL & ENFERMERÍA & POLITÉCNICA \\
\hline Enfermo (53.4\%) & Infeliz (66.1\%) & Enfermo (48.0\%) \\
Infeliz $(51.1 \%)$ & Enfermo $(59.7 \%)$ & Infeliz (48.0\%) \\
Delincuente (27.3\%) & Delincuente (45.2\%) & Delincuente (40.0\%) \\
Víctima (21.6\%) & Inconformista (12.9\%) & Inconformista (14.0\%) \\
\hline
\end{tabular}

Los datos segmentando la muestra de estudiantes de Trabajo Social son:

Tabla 3: Adjetivos diferenciales (en \%).

\begin{tabular}{|l|r|r|r|}
\hline & $1^{\circ} \mathrm{TS}$ & $3^{\circ} \mathrm{TS}$ & demás \\
\hline Víctima & 22.2 & 20.9 & 5.4 \\
\hline Delincuente & 28.9 & 25.6 & 42.9 \\
\hline
\end{tabular}

Aparece claramente reflejada la diferencia de los estudiantes de Trabajo Social desde el momento de ingreso en el Centro, lo que podría implicar características grupales diferenciales del resto de los estudiantes.

Así pues, podemos concluir que se confirma la influencia del contexto en el conocimiento prototípico como una construcción social en función de las ideas personales.

\subsection{Actitud.}

Una actitud implica una asociación entre un objeto social y una evaluación determinada. Tradicionalmente se analiza en función de sus tres componentes: cognitivo, afectivo y conductual. 


\subsubsection{Componente conductual:}

Es la tendencia, disposición o intención de conducta hacia un objeto.

La pregunta del cuestionario estaba planteada con sentido negativo, es decir, se les preguntaba sobre la conducta que les disgustaría realizar, por lo que los porcentajes deben interpretarse en dirección contraria, cuanto más bajo es su valor supone mayor acercamiento conductual.

Los resultados obtenidos han sido:

Tabla 4: Cosas que te disgustaría realizar con una persona violenta (en \%).

\begin{tabular}{|l|c|c|c|}
\hline & Trabajo Social & Enfermería & Politécnica \\
\hline Estudiar juntos & 9.1 & 6.5 & 18.0 \\
\hline Vivir en el mismo sitio & 35.2 & 32.3 & 60.0 \\
\hline Salir en el mismo grupo & 14.8 & 8.1 & 10.0 \\
\hline Ser amigos & 9.1 & 8.1 & 6.0 \\
\hline Que saliera con mi hermano/a & 29.5 & 50.0 & 26.0 \\
\hline Casarme con él/ella & 63.6 & 88.7 & 34.0 \\
\hline Ninguna & 11.4 & 1.6 & 20.0 \\
\hline NS/NC & 5.7 & 0 & 0 \\
\hline
\end{tabular}

Estos resultados suponen una mayor distancia social en la muestra de Enfermería, y más proximidad en los alumnos de la Politécnica, posicionándose los alumnos de Trabajo Social entre ambos. Estos mismos resultados aparecen en los datos con la muestra de Trabajo Social segmentada.

Los porcentajes más altos en Trabajo Social y Enfermería se producen en la categoría "casarse con él/ella" (hasta casi el $89 \%$ en Enfermería), frente a un $34 \%$ en la muestra procedente de la Politécnica, dirigiéndose el mayor porcentaje en estos últimos hacia "vivir en el mismo sitio".

Estos resultados no pueden ser explicados desde la hipótesis de partida, ante la discordancia surgida y teniendo en cuenta la diferente distribución de las muestras en función del sexo, procedimos a segmentar la muestra (en su globalidad) en función de esta variable para intentar dar respuesta a los resultados encontrados.

Los porcentajes de respuesta de la categoría "vivir en el mismo sitio" fueron $33.6 \%$ mujeres y $52.9 \%$ de los hombres; en "casarme con él/ella" fueron: $79.4 \%$ de mujeres y $35.3 \%$ en el caso de los varones.

En la gráfica 4 se reproducen los sectores de respuesta para la categoría "casarse" por sexos, ya que es en la que se producen las mayores diferencias. 
Gráfica 4: Porcentajes de respuesta "casarme con él/ella".

SEXO: 1 mujer

Si tenemos en cuenta que la muestra de la Politécnica está constituida mayoritariamente por hombres, podría ser que la diferencia de sexos explicara la variabilidad intergrupal encontrada. Esta hipótesis queda confirmada con los resultados de la muestra segmentada por sexos y Centros.

Con estos resultados podemos concluir que la conducta parece estar más vinculada al sexo que al Centro de estudio.

\subsubsection{Componente afectivo:}

Está constituido por los sentimientos que dicho objeto despierta.

Los resultados obtenidos han sido:

Tabla 5: ¿Qué reacción te producen las personas violentas? (en \%).

\begin{tabular}{|l|c|c|c|}
\hline & Trabajo Social & Enfermería & Politécnica \\
\hline Miedo & 34.1 & 37.1 & 22.0 \\
\hline Rechazo & 20.5 & 37.1 & 40.0 \\
\hline Ayuda & 23.9 & 4.8 & 6.0 \\
\hline Indiferencia & 1.1 & 4.8 & 12.0 \\
\hline Lástima & 15.9 & 8.1 & 20.0 \\
\hline
\end{tabular}

En la tabla 5 y la gráfica 5 aparecen conjuntamente reflejados los porcentajes de respuesta de la variable referida al componente afectivo actitudinal para los tres Centros. El perfil que aparece por Centros es:

- Enfermería: Hay un predominio de miedo y rechazo, estando a mucha distancia las demás reacciones.

- Trabajo Social: Predominio también de miedo, rechazo en menor porcentaje y aparece ayuda como elemento diferenciador de grupo. 


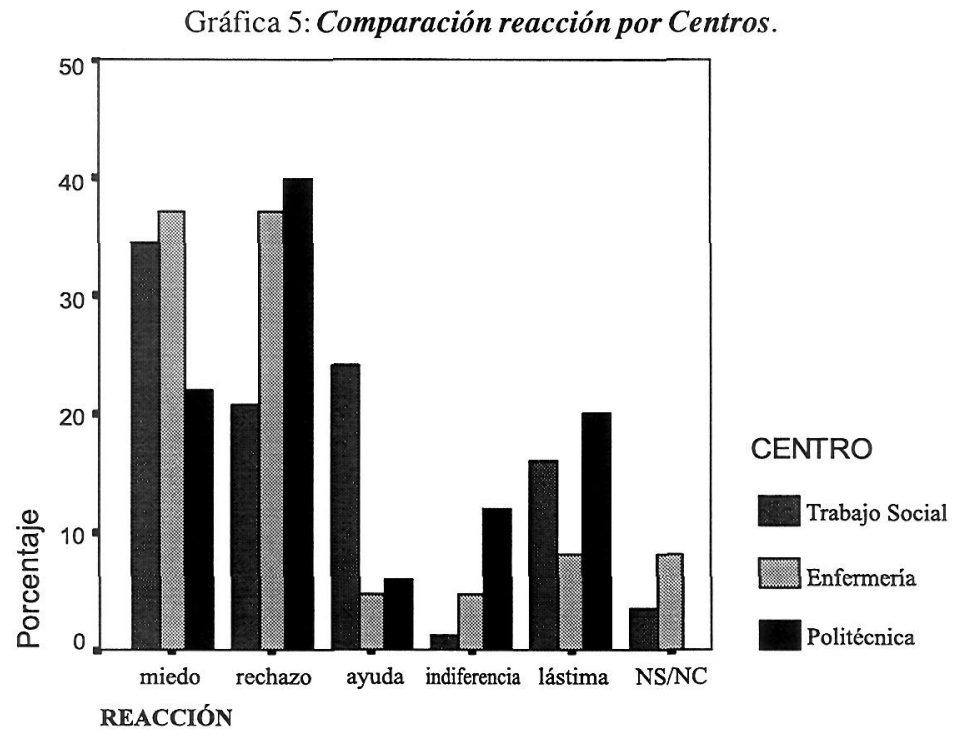

- Politécnica: se altera el orden de los términos, en primer lugar rechazo y a distancia miedo e indiferencia.

$\mathrm{Si}$ analizamos la contingencia de respuesta con el sexo encontramos una alta vinculación, el $81 \%$ de los sujetos que afirman tener "miedo" son mujeres, y el 90\% de los que marcan "indiferencia" son varones. Asociación que se mantiene en la segmentación de la muestra. Probablemente el miedo predominante en Trabajo Social y Enfermería lleve al incremento de la respuesta "casarse", mientras que el rechazo origine la respuesta de "vivir en el mismo sitio", produciéndose con ello una correlación de respuesta entre el componente conductual y afectivo.

En cuanto a la respuesta "ayuda" aparece con un porcentaje mucho más elevado en Trabajo Social, suponiendo casi el $80 \%$ de esta categoría. Aparece asociada a la variable sexo, mujeres $16.8 \%$ y varones $5.9 \%$, pero tras segmentar conjuntamente Centro y sexo la diferencia aparece asociada a estudio, dándose los siguientes porcentajes de ayuda en las mujeres de cada muestra: Trabajo Social $-29.2 \%$, Enfermería $-3.9 \%$ - y Politécnica -7.1\%-. Así pues parece como reacción específica de Trabajo Social. Datos que se confirman con la segmentación de la muestra de Trabajo Social, ya que aparece claramente reflejada la diferencia con los alumnos de primer curso, observándose un incremento en los alumnos de tercer curso, posiblemente fruto de la formación recibida. También se observa disminución de la respuesta "rechazo" y desaparición de la categoría "indiferencia". Datos que quedan reflejados en la tabla 6. 
Tabla 6: Componente afectivo actitudinal, muestra segmentada .

\begin{tabular}{|l|r|r|r|}
\hline & $1^{\circ} \mathrm{TS}$ & $3^{\circ} \mathrm{TS}$ & demás \\
\hline Rechazo & 26.7 & 14.0 & 38.4 \\
\hline Ayuda & 20.0 & 27.9 & 5.4 \\
\hline Indiferencia & 2.2 & 0.0 & 8.0 \\
\hline
\end{tabular}

Así pues, los sentimientos que despierta una persona violenta son distintos para los sujetos de las diferentes muestras. Es común el miedo y el rechazo, aunque el rechazo es menor en los alumnos de Trabajo Social y además se reduce tras los tres años de estudio. Y con carácter diferencial en Trabajo Social la "ayuda" que aparece claramente como sentimiento de grupo, siendo también más elevada al final de la carrera de Trabajo Social. Diferencias que aparecen vinculadas significativamente $(p=0.00)$ con la variable Centro.

\subsubsection{Componente cognitivo:}

Compuesto por las cogniciones, ideas, de la persona sobre el objeto de la actitud.

Los resultados obtenidos han sido:

Tabla 7: Las personas violentas son: (en \%).

\begin{tabular}{|l|c|c|c|}
\hline & Trabajo Social & Enfermería & Politécnica \\
\hline Víctimas de situaciones & 25.0 & 12.9 & 16.0 \\
\hline Asociales & 8.0 & 12.9 & 20.0 \\
\hline Víctimas de una enfermedad & 23.9 & 22.6 & 18.0 \\
\hline Delincuentes & 3.4 & 21.0 & 12.0 \\
\hline Personas comientes, que tienen ese problema & 33.0 & 25.8 & 28.0 \\
\hline
\end{tabular}

En la tabla 7 y gráfica 6 se aprecia que la mayor diferencia se da en la respuesta "delincuentes", siendo muy inferior el porcentaje en los alumnos de Trabajo Social. También aparece diferencia en "son víctimas de situaciones", considerando esta alternativa en mayor medida los alumnos de Trabajo Social.

Podemos agrupar las alternativas de respuesta en dos categorías en función de su referencia a factores externos -factor situacional-o a factores internos -factor disposicional-. Constituyen factores situacionales: son víctimas de situaciones, son víctimas de una enfermedad y son personas corrientes que tienen ese problema. El factor disposicional está formado por la suma de: son asociales y son delincuentes. 


\section{Gráfica 6: Componente cognitivo, por Centros.}

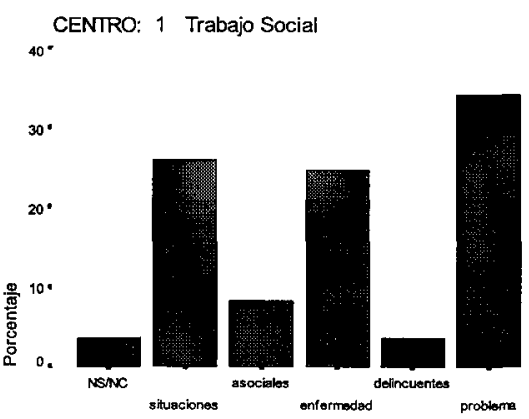

PERSONAS

CENTRO: 2 Enfermer a

$$
30^{\circ}
$$

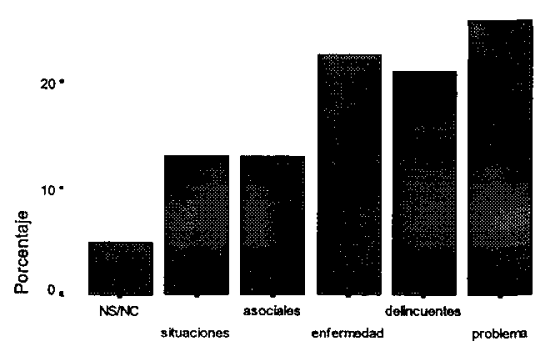

PERSONAS

CENTRO: 3 Polit cnica

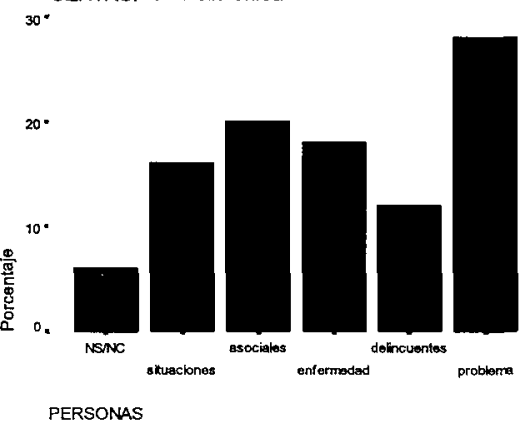

Tras esta agrupación de respuestas aparecen los siguientes resultados:

Tabla 8: Factores componente cognitivo (en \%).

\begin{tabular}{|l|c|c|c|}
\hline & Trabajo Social & Enfermería & Politécnica \\
\hline F. Disposicional & 11.4 & 33.9 & 32.0 \\
\hline F. Situacional & 81.9 & 61.3 & 62.0 \\
\hline
\end{tabular}


Se produce coincidencia en la concepción de la violencia como ocasionada fundamentalmente por factores situacionales. Pero los datos reflejan también un componente diferencial cuantitativo, es mucho más elevado el factor situacional en los alumnos de Trabajo Social.

Diferencia que aparece ya en primer curso de Trabajo Social, tal y como queda reflejado en el siguiente gráfico.

\section{Gráfica 7: Factores componente cognitivo.}

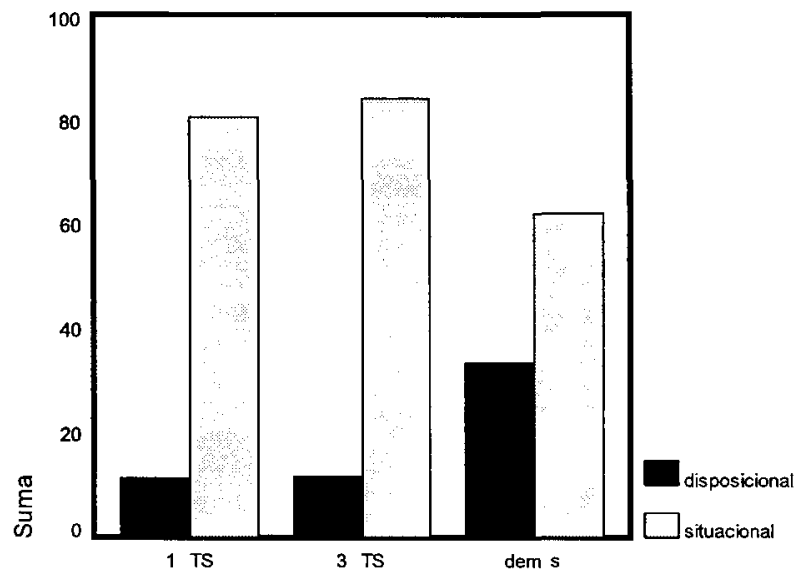

GRUPO

Podemos concluir que se reproducen los datos de los apartados anteriores, clara influencia de la educación social pero con un componente diferencial de grupo en los estudiantes de Trabajo Social.

Pasamos a analizar la consistencia afectivo-conativa, es decir, el grado en el que el componente afectivo y el cognitivo son contingentes. La vinculación es significativa al nivel del $95 \%$, produciéndose en la siguiente dirección:

- El 44\% de los sujetos que conceptualizan como asociales presentan una reacción de rechazo.

- El 45.5\% que considera que la persona violenta es víctima de una enfermedad reacciona con miedo.

- El $59.1 \%$ de los que consideran que son delincuentes actúan con rechazo.

- También parece significativo que el $55 \%$ de los que no contestan a la pregunta referida al componente cognitivo responden afectivamente con rechazo.

- Los sujetos cuya reacción es de ayuda se distribuyen en el compo- 
nente cognitivo: $26 \%$ situaciones, $30 \%$ enfermedad, $41 \%$ problema y $0 \%$ delincuentes. Que coincide con las respuestas que aparecen en mayor porcentaje en los alumnos de Trabajo Social.

\section{CONCLUSIÓN.}

Los resultados de nuestro estudio reflejan un elevado grado de acuerdo general entre los estudiantes de los tres Centros, apoyando la influencia del contexto sociocultural donde se encuentran inmersos.

Esta homogeneidad de respuesta va unida a peculiaridades específicas de los alumnos de Trabajo Social, que los define como más abiertos, tolerantes, hacia las personas violentas. Poseyendo características de grupo que les hacen diferentes en la visión del problema de violencia. Estas características de grupo son fundamentalmente dirigidas hacia una consideración del problema de agresión como factor social, situándose abiertamente en una posición de "ayuda".

Aparece también reflejado el componente formativo como un incremento de las características iniciales de grupo y con una más elevada aceptación a nivel actitudinal de las personas violentas. 